\title{
HISTORY OF HYDROGEN REIONIZATION IN THE COLD DARK MATTER MODEL
}

\author{
Christopher A. OnKen and Jordi Miralda-Escudé \\ Department of Astronomy, Ohio State University, \\ 140 West 18th Street, Columbus, OH 43210-1173; onken@astronomy.ohio-state.edu, jordi@astronomy.ohio-state.edu \\ Received 2003 July 9; accepted 2004 March 28
}

\begin{abstract}
We calculate the reionization history in CDM (cold dark matter) models. The epoch of the end of reionization and the Thomson scattering optical depth to the cosmic microwave background depend on the power spectrum amplitude on small scales and on the ionizing photon emissivity per unit mass in collapsed halos. We calibrate the emissivity to reproduce the measured ionizing background intensity at $z=4$. Models in which all CDM halos have either a constant emissivity or a constant energy emitted per Hubble time per unit mass predict that reionization ends near $z \sim 6$ and that the optical depth is in the range $0.05<\tau_{e}<0.09$, consistent with Wilkinson Microwave Anisotropy Probe (WMAP) results at the 1-2 $\sigma$ level. If the optical depth is as high as 0.17 (as suggested by $W M A P$ ), halos of velocity dispersion $\sim 3-35 \mathrm{~km} \mathrm{~s}^{-1}$ at $z>15$ must have ionizing emissivities per unit mass larger by a factor $\gtrsim 50$ compared with the more massive halos that produce the ionizing emissivity at $z=4$. This factor increases to 100 if the CDM power spectrum amplitude is required to agree with the recent Croft et al. measurement from the Ly $\alpha$ forest. If $\tau_{e} \gtrsim 0.17$ is confirmed, a higher ionizing emissivity at $z>15$ compared with $z=4$ might arise from an enhanced star formation rate or quasar abundance per unit mass and an increased escape fraction for ionizing photons; the end of reionization could have been delayed to $z \sim 6$ because of the suppression of gas accretion and star formation in low-mass halos as the medium was reionized.
\end{abstract}

Subject headings: cosmology: theory — diffuse radiation — galaxies: formation — intergalactic medium

\section{INTRODUCTION}

The epoch of reionization of the universe started with the emission of the first ionizing photons into the intergalactic medium (IGM), and ended when all the low-density regions of the universe were ionized. During this period, the number of ionizing photons reaching the IGM had to be sufficient to ionize every atom in the universe and to balance any recombinations in the IGM (in addition, many more ionizing photons may have been emitted in regions of dense self-shielded gas, which were locally absorbed and did not contribute to ionizing the IGM). At the end of reionization, the mean free path of ionizing photons increased to scales much larger than the typical separation of the collapsed halos hosting the sources. Regions of dense self-shielded gas (observed as Lyman limit systems) shrank in size as the ionizing background increased in intensity, because of the growing mean free path (MiraldaEscudé et al. 2000). If the fraction of baryons in regions of intermediate density that were ionized as the mean free path increased was small, the rise in the background intensity could be relatively fast, as seen in the numerical simulations of Gnedin (2000).

Precisely this rapid change of the ionizing background intensity has been inferred by Fan et al. (2002) to occur at $z \simeq 6$, by analyzing the change of the mean transmitted flux of the Ly $\alpha$ forest in the highest redshift quasars known. The transmitted flux apparently drops abruptly to very low levels near $z=6$. This observation most likely implies that the end of reionization occurred, in fact, at $z \simeq 6$, although as a larger number of quasars at $z>6$ are discovered, more detailed investigations of this issue will need to be done to understand precisely how the mean free path grew. In particular, the number of small gaps of transmitted flux, such as the one seen in a $z=6.37$ quasar by White et al. (2003), provides information on the size of the $\mathrm{H}$ II regions before they overlapped (Miralda-Escudé 1998; Barkana 2002).

The results of the Wilkinson Microwave Anisotropy Probe (WMAP; Bennett et al. 2003 and references therein) have strengthened the observational support of the cold dark matter (CDM) scenario in a flat model that contains a vacuum energy component (in addition to the ordinary baryonic matter and dark matter). The model parameters are being measured with increasing accuracy via combined observations of the cosmic microwave background (CMB), galaxy clustering, weak lensing, and the $\operatorname{Ly} \alpha$ forest. The theory then makes an increasingly reliable prediction for the abundance of collapsed dark matter halos as a function of mass and redshift. In these halos the process of radiative gas dissipation and star formation can take place, leading to the emission of ionizing radiation. However, the uncertainties in the efficiency of star formation, the stellar initial mass function (IMF), the emission due to gas accretion into black holes (be they in X-ray binaries or active galactic nuclei), and the fraction of the ionizing photons that escape into the IGM from the dense regions in which they are emitted prevent a clear prediction for the history of reionization.

Even if the time at which reionization ends is conclusively determined to be at $z \simeq 6$ by the Gunn-Peterson trough (Gunn \& Peterson 1965) observations, there may have been a long interval of time in which the universe was partially ionized, with cosmological $\mathrm{H}$ II regions around sources filling only some fraction of the volume. A very good observational probe for the characteristic time at which the bulk of the baryons in the universe were ionized is the electron-scattering optical depth to the CMB, $\tau_{e}$. This was measured for the first time by WMAP (Kogut et al. 2003; Spergel et al. 2003) using the large-scale polarization-temperature anisotropy correlation. At present, the result is still subject to a large error, both observational and model dependent: Kogut et al. find a model-independent value 
of $\tau_{e}=0.16 \pm 0.04$ from the polarization-temperature correlation of the WMAP data, but Spergel et al. find $\tau_{e}=0.17 \pm$ 0.06 from a fit of a CDM model with a running spectral index to a combined data set of $\mathrm{CMB}$, galaxy clustering, and $\mathrm{Ly} \alpha$ forest observations and $\tau_{e}=0.12 \pm 0.06$ when the fit is forced to the usual CDM model with a primordial power-law spectrum. A value of $\tau_{e}=0.17$ would imply that most baryons were reionized as early as $z \sim 20$.

The announcement of the measurement of $\tau_{e}$ by $W M A P$ has led to a large number of papers discussing the possibility of this early reionization in the CDM model (Wyithe \& Loeb 2003; Haiman \& Holder 2003; Holder et al. 2003; Ciardi et al. 2003; Somerville \& Livio 2003; Sokasian et al. 2003; Cen 2003; Chiu et al. 2003). These authors have generally found that if one is willing to assume a high efficiency in the production of ionizing photons per unit mass in the low-mass halos collapsing at early times, then early reionization and a high optical depth are possible in CDM models. Naturally, there are fewer halos massive enough to form stars at high redshift when the power spectrum amplitude on small scales is lower, so the required efficiency is higher, and some models can be ruled out on the basis of their small power on small scales, such as the warm dark matter model (Spergel et al. 2003). But to a large extent, the ability of the CDM model to accommodate a wide range for the epoch at which the bulk of the IGM was reionized simply reflects our ignorance of the efficiency in producing ionizing photons that escape to the IGM.

However, a reasonable question we can ask is how the required efficiency compares with any observationally determined values of the rate at which ionizing photons have been emitted over the history of the universe, taking into account the predicted abundances of halos of different masses that form at each redshift. In the work we present here, the ionizing emissivity is calibrated to the observations of the ionizing background intensity at $z=4$. The basic idea is that we know approximately the abundance of halos as a function of mass that existed at $z=4$ and the global rate at which ionizing photons were emitted at that epoch. The most reasonable assumption to make seems to be that we have a similar emissivity in similar types of halos at each redshift. If this is not sufficient to reionize the universe by a certain epoch or to produce a certain value of the Thomson optical depth to the CMB, we can then see by what factor the emissivity must be increased to achieve the desired result.

We base the calibration of the ionizing emissivity at $z=4$ on observations of the abundance of Lyman limit systems and of the Ly $\alpha$ forest, from which the ionizing background intensity and global emissivity are derived as described in MiraldaEscudé (2003). The Lyman limit systems dominate the absorption of ionizing radiation, and their abundance determines directly the mean free path of ionizing photons. The Lyman limit system abundance can be reasonably well determined up to $z=4$ (Storrie-Lombardi et al. 1996; at higher redshift the increasing opacity of the forest of Lyman series lines makes it increasingly difficult to identify Lyman limit systems); hence, we choose this redshift as our calibration point. The intensity of the ionizing background is obtained from the measured mean transmitted flux of the Ly $\alpha$ forest, combined with CDM simulations of $\operatorname{Ly} \alpha$ forest spectra and independent measurements of the baryon density of the universe. Then, the ratio of the background intensity to the mean free path yields the volume-averaged emissivity. This calculation shows that the maximum allowed emissivity consistent with the present observational determinations is 7 ionizing photons per baryon per
Hubble time at $z=4$ (Miralda-Escudé 2003). Remaining uncertainties on the value of this emissivity are related mainly to the temperature of the IGM and the CDM power spectrum amplitude.

The key question for reionization, of course, is how this emissivity determined at $z=4$ changes with increasing redshift. If the emissivity per unit comoving volume stayed constant at $z>4$ (irrespective of the evolving halo population), the optical depth $\tau_{e}$ would not be larger than 0.09 (Miralda-Escudé 2003). In CDM models, the fraction of mass contained in halos in which stars can form decreases with redshift at $z>4$, so this implies that the condition $\tau_{e}>0.09$ (which requires an increase of the emissivity with redshift) requires an increasing efficiency with redshift in emitting ionizing photons from collapsed halos.

In this paper we adopt a very simple model for how the ionizing emissivity depends on halo mass and redshift that still captures the essential physical ingredient for the process of reionization: as the medium is ionized, the gas is heated and the Jeans mass is raised, implying that some low-mass halos where gas can collapse when the medium is neutral are unable to continue to accrete gas after the medium is ionized. We divide halos into two populations, the "high-mass" ones (population A) that are able to accrete gas and form new stars irrespective of the state of the IGM and the "low-mass" ones (population B) that can form stars and emit ionizing radiation only when the medium is neutral. Low-mass halos collapse first in CDM, but as they ionize the medium around them the rate of star formation is suppressed, until the high-mass halos start to collapse (Couchman \& Rees 1986; Shapiro et al. 1994). A similar separation of halo classes was made by Haiman \& Holder (2003); our population A corresponds to their type II and type Ia halos (Haiman \& Holder separated these halos into the ones that can or cannot cool by atomic cooling after the accreted gas has reached the typical overdensity of $\sim 200$ at virialization in the absence of any initial entropy; we do not distinguish between these two classes in our more simple model), and our population B corresponds to their type Ib population.

We describe our model for the efficiency to emit ionizing radiation in detail in $\S 2$. Results are presented in $\S 3$, and a general discussion is given in $\S 4$.

\section{MODELS}

\subsection{CDM Power Spectrum}

We use three CDM models to predict the halo abundances. All the models assume flat space with a cosmological constant, with $\Omega_{m 0}=0.257, \Omega_{b 0}=0.0436, h=0.72$, and a power-law primordial power spectrum. These parameters are based on the power-law CDM model fit of Spergel et al. (2003; see their Table 7). The three models vary in the normalization and slope of the power spectrum: model 1 has $\sigma_{8}=0.81$ and $n_{s}=0.96$; model 2 has $\sigma_{8}=0.85$ and $n_{s}=0.98$; and model 3 has $\sigma_{8}=$ 0.89 and $n_{s}=1.00$ (where $\sigma_{8}$ is the linear rms mass fluctuation at present on spheres of radius $8 h^{-1} \mathrm{Mpc}$ and $n_{s}$ is the primordial scalar spectral index). The power spectrum is computed with the fitting formula of Eisenstein \& $\mathrm{Hu}$ (1999). Model 1 has the smallest amplitude of fluctuations on the small scales at which the first halos that emit ionizing radiation collapse $(0.03-1 \mathrm{Mpc})$, and model 3 has the largest amplitude. Model 3, with the largest power on small scales, has halos forming at the highest redshift and therefore predicts an earlier start of reionization and higher optical depth, 


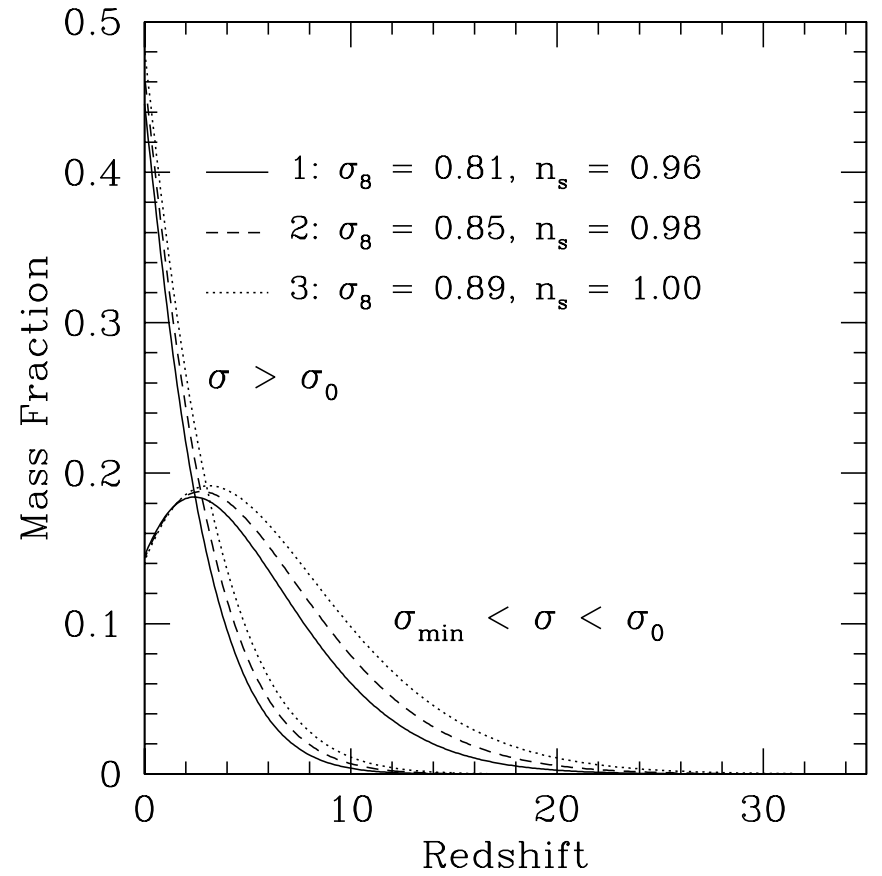

FIG. 1.-Fraction of mass collapsed into halos as a function of redshift for two ranges in halo velocity dispersion, $\sigma$. Population A includes the halos with $\sigma>\sigma_{0}$, while population B represents the halos with $\sigma_{\min }<\sigma<\sigma_{0}$. For each of the model 1 (solid lines), model 2 (dashed lines), and model 3 (dotted lines) cosmological parameter sets, $\sigma_{\min }=3.68 \mathrm{~km} \mathrm{~s}^{-1}$ and $\sigma_{0}=35 \mathrm{~km} \mathrm{~s}^{-1}$.

$\tau_{e}$, than the other models for a fixed ionizing emissivity per unit mass.

Model 1 has the same parameters as the best-fit power-law model found by WMAP (Bennett et al. 2003; Spergel et al. 2003 ) to the combined data set of CMB observations, the TwoDegree Field Galaxy Redshift Survey (Colless et al. 2001), and Ly $\alpha$ forest power spectrum data (Croft et al. 2002). We do not include the running spectral index introduced in Spergel et al.; inclusion of the running spectral index reduces the power on small scales, making it far more difficult to have enough collapsed halos at high redshift to obtain an early reionization (Yoshida et al. 2003b). Models 2 and 3 raise $\sigma_{8}$ and $n_{s}$ by 1 and $2 \sigma$, respectively, above the best-fit power-law model. Increasing these parameters produces additional power on small scales and more halos at higher redshift.

Measurement of the Ly $\alpha$ forest has allowed a determination of the power spectrum amplitude on the smallest scales. We therefore compare the amplitude of our models with the determination of the Ly $\alpha$ forest power spectrum by Croft et al. (2002), who find a normalization at redshift $z=2.72$ of

$$
\Delta^{2}\left(k_{p}\right) \equiv \frac{k_{p}^{3}}{2 \pi^{2}} P\left(k_{p}\right)=0.74_{-0.16}^{+0.20}
$$

where $k_{p}=0.03\left(\mathrm{~km} \mathrm{~s}^{-1}\right)^{-1}$ [we use the convention of multiplying all distances by the Hubble constant $H(z)$ at the redshift at which we measure the power spectrum, and the wavenumbers by $H^{-1}(z)$ to express them in velocity units]. The normalization of our models 1,2 , and 3 is $\Delta^{2}\left(k_{p}\right)=$ $0.89,1.04$, and 1.21, respectively. Hence, models 2 and 3 have a power spectrum amplitude higher than the Croft et al. (2002) measurement by 1.5 and $2.3 \sigma$, respectively. We note, however, that the quoted error in equation (1) does not include some potentially important systematic effects, as discussed by Croft et al. In fact, Seljak et al. (2003) argued for a higher value

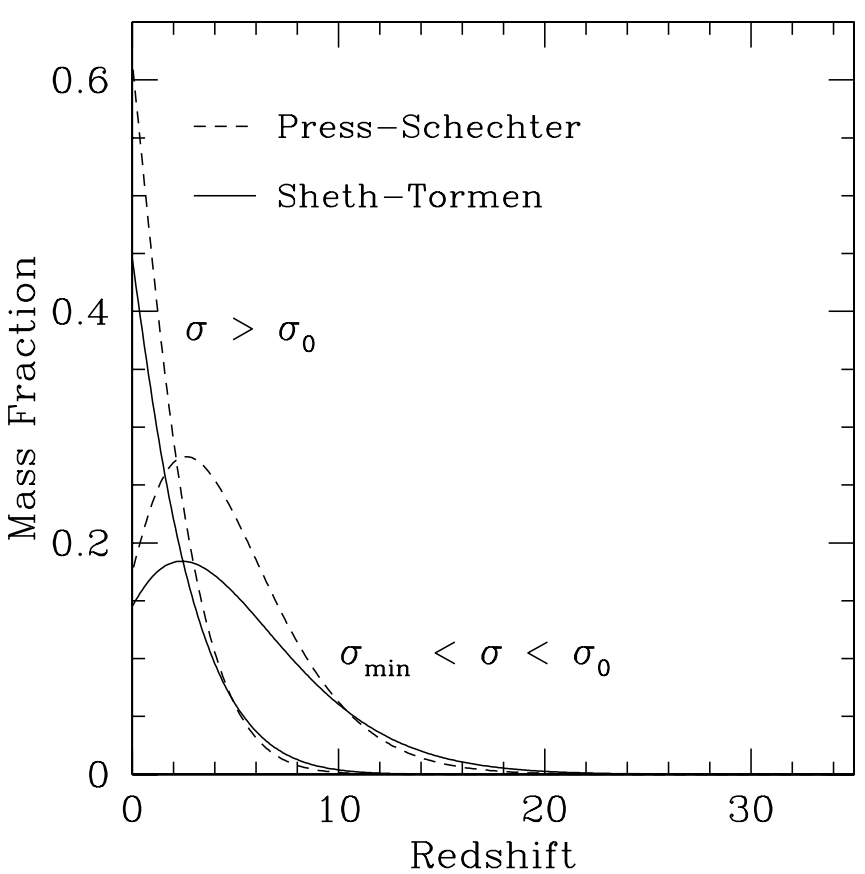

FIG. 2.-Comparison of the amount of collapsed mass in halo populations $\mathrm{A}$ and $\mathrm{B}$ as a function of redshift, derived with the Sheth-Tormen (solid lines) and Press-Schechter (dashed lines) mass functions for model 1.

of the mean transmitted flux than assumed in Croft et al. and an implied power spectrum amplitude derived from the Ly $\alpha$ forest that is higher and has a larger error than that allowed by Croft et al.; in this case, model 3 would not be less favored by the data compared with model 1.

\subsection{Halo Populations}

We use the Sheth \& Tormen (1999) prescription for computing the abundances of halos as a function of mass and redshift. Halos are divided into two populations: those that do not emit any ionizing photons after the medium around them has been reionized (population B) and those that are massive enough to continue forming stars and producing ionizing photons after reionization (population A). In this paper we place the division between the two populations at a velocity dispersion $\sigma_{0}=35 \mathrm{~km} \mathrm{~s}^{-1}$, on the basis of the suppression of gas infall and cooling in smaller halos caused by photoionization (Efstathiou 1992; Thoul \& Weinberg 1996; Navarro \& Steinmetz 1997). The lower limit to the velocity dispersion of the population B (low mass) halos is placed at $\sigma_{\min }=3.68 \mathrm{~km}$ $\mathrm{s}^{-1}$ because molecular hydrogen cooling below a temperature of $\sim 2000 \mathrm{~K}$ is ineffective (Yoshida et al. 2003a).

The fraction of baryons, $F_{A, B}$, that occupy each of the halo populations is shown in Figure 1 for models $1-3$. We also show in Figure 2 the difference between using the ShethTormen and the Press-Schechter (Press \& Schechter 1974) mass functions for model 1. The Sheth-Tormen mass function predicts a slightly larger number of objects at the highest redshifts.

\subsection{Emissivity}

For population A, i.e., halos with $\sigma>\sigma_{0}=35 \mathrm{~km} \mathrm{~s}^{-1}$, the ionizing photon emissivity per baryon is assumed to be of the form

$$
\epsilon_{A}(z)=F_{A}(z) \frac{\epsilon_{4}}{F_{A}(4)}\left(\frac{1+z}{5}\right)^{\alpha}
$$


where $F_{A}(z)$ is the fraction of baryons in population A halos at redshift $z$ and $\epsilon_{4}$ is the total emissivity at $z=4$, which is assumed to be equal to 7 ionizing photons per baryon per Hubble time at $z=4$, the upper limit obtained in MiraldaEscudé (2003). We use two values of $\alpha$ in this paper: for $\alpha=0$ the energy emitted per unit physical time per unit mass remains constant, and for $\alpha=1.5$ the total energy emitted per Hubble time at redshift $z$ remains constant. Note that gas cooling is inefficient in very massive halos when the virialized gas temperature is higher than $10^{7} \mathrm{~K}$, implying that star formation efficiency in very massive halos (similar to present clusters of galaxies) should be reduced. However, at $z=4$ the abundance of these halos, which arise from fluctuations larger than $4 \sigma$, is negligibly small.

For population B, we allow an emissivity per unit mass different from the one in population A halos, parameterizing it as

$$
\epsilon(\sigma)=\left(1+\gamma \ln \frac{\sigma_{0}}{\sigma}\right) \frac{\epsilon_{4}}{F_{A}(4)}\left(\frac{1+z}{5}\right)^{\alpha}
$$

where $\gamma$ is a scaling factor used to adjust the variation in efficiency between the low-mass and high-mass halos. This results in a smooth variation of the emissivity with $\sigma$. The population $\mathrm{B}$ emissivity is then

$$
\epsilon_{B}(z)=\int_{\sigma_{\min }}^{\sigma_{0}} f_{B}(\sigma, z) \epsilon(\sigma) d \sigma
$$

where $f_{B}$ is the fraction of mass in halos in the interval $d \sigma$ around $\sigma$, and we set $\sigma_{\min }=3.68 \mathrm{~km} \mathrm{~s}^{-1}$, corresponding to $2000 \mathrm{~K}$ for the mean particle mass of the primordial atomic gas.

\subsection{Analytical Framework}

The equation for the ionization of the IGM is

$$
\begin{aligned}
\frac{d y}{d t} & =\epsilon-R y \\
& =\epsilon_{A}+\epsilon_{B}(1-y)-R y
\end{aligned}
$$

where $y$ is the volume fraction of the IGM that is ionized at any given time $t$ and $R$ is the mean number of recombinations per baryon in the ionized regions per unit time (Madau et al. 1999; Miralda-Escudé 2003). This equation makes the approximation that the photon mean free path is very short, so emitted photons are instantaneously used to ionize atoms. It also hides all the complicating physics of reionization in the averaged recombination rate $R$, which depends on the clumping factor of the photoionized gas. Note that population $\mathrm{B}$ halos emit ionizing radiation only when they are located in the atomic medium, so the emissivity $\epsilon_{B}$ is multiplied by $1-y$. We assume a clumping factor of unity throughout this paper, computing the recombination coefficient at $T=10^{4} \mathrm{~K}$, which gives $R=1.16 \times 10^{-17} \mathrm{~s}^{-1}$ at $z=4$ [note that $R$ is proportional to the density and therefore to $(1+z)^{3}$ when the clumping factor is constant]. The solution of equation (6) is

$$
\begin{aligned}
y(t)= & \int_{t_{i}}^{t} \exp \left\{-\int_{t^{\prime}}^{t}\left[R\left(t^{\prime \prime}\right)+\epsilon_{B}\left(t^{\prime \prime}\right)\right] d t^{\prime \prime}\right\} \\
& \times\left[\epsilon_{B}\left(t^{\prime}\right)+\epsilon_{A}\left(t^{\prime}\right)\right] d t^{\prime}
\end{aligned}
$$

where $t_{i}$ is an arbitrarily chosen initial time (before any appreciable halo formation).

\subsection{Optical Depth}

The Thomson scattering optical depth, $\tau_{e}$, is

$$
\tau_{e}(z)=\frac{n_{e} \sigma_{e} c}{H_{0}} \int_{0}^{z} \frac{F_{i}(1+z)^{2} y(z) d z}{\left[\Omega_{\Lambda 0}+\Omega_{m 0}(1+z)^{3}\right]^{1 / 2}},
$$

where $\Omega_{\Lambda 0}=1-\Omega_{m 0}, n_{e}$ is the comoving electron number density, $\sigma_{e}$ is the electron cross section, and $F_{i}$ is the fraction of the baryons in the ionized regions that are actually in the ionized IGM (with the rest being in self-shielded atomic or molecular clouds or in stars). We assume $F_{i}=0.9$ (as in Miralda-Escudé 2003) for $z<6$, but $F_{i}=1$ at $z>6$; lowering $F_{i}$ at $z>6$ would demand starting reionization at higher redshift to obtain a fixed optical depth, increasing the required emissivity from the first star-forming halos (note that eq. [5] needs to be modified if $F_{i}<1$ during reionization). For our value of $\Omega_{b} h^{2}=0.023$, the electron number density is $n_{e}=$ $2.7 \times 10^{-7}\left(1-f_{Y} Y\right) \mathrm{cm}^{-3}$, where $Y=0.24$ is the helium abundance by mass and $f_{Y}=0.5$ and 0.75 when the helium is doubly and singly ionized, respectively. We assume that helium becomes doubly ionized at $z=3.5$ (e.g., Heap et al. 2000) and that it is singly ionized at higher redshift wherever hydrogen is ionized. This results in an optical depth up to $z=6$ of $\tau_{e}(z=6)=0.032$.

\section{RESULTS}

We now present the results on the fraction of the volume that is ionized as a function of redshift, $y(z)$, with the emissivity that arises from our two populations of halos given by equations (2) and (3). To summarize the emissivity model, the population A (high-mass halos) emissivity per unit mass is fixed at $z=4$ and is allowed to vary as $(1+z)^{\alpha}$, while the population $\mathrm{B}$ (low-mass halos) emissivity per unit mass can also be increased with the parameter $\gamma$.

Figure 3 shows $y(z)$ for each of the three CDM models and for both $\alpha=0$ and 1.5. The case $\gamma=0$, in which the emissivity per unit mass from populations $\mathrm{A}$ and $\mathrm{B}$ is the same, is shown as solid lines. The cases $\gamma=1,10$, and 100 are shown as dotted lines, dashed lines, and dash-dotted lines, respectively. The models with high $\gamma$-values have an early reionization as the first population $\mathrm{B}$ halos collapse; then, $y(z)$ grows slowly when it approaches unity because of the decrease in the emission from population $B$ as most of the IGM is ionized, and finally, $y$ reaches unity when the population A halos start forming and emitting radiation in the ionized regions of the universe. The optical depths to electron scattering for all the models shown are listed in Table 1.

The emissivity as a function of redshift is shown in Figure 4 for the same models. At redshift just above 4, the emissivity declines with redshift because the abundance of population A halos (which are the only ones contributing when the medium is entirely ionized) drops rapidly. The emissivity increases again at redshifts higher than at the end of reionization, when population B halos contribute. Models with higher $\gamma$ can obviously have a higher emissivity at very high redshift, but their emissivity declines at intermediate redshifts when $y$ is close to unity and the emission from population $\mathrm{B}$ halos is suppressed, as assumed in equation (3). The models with 


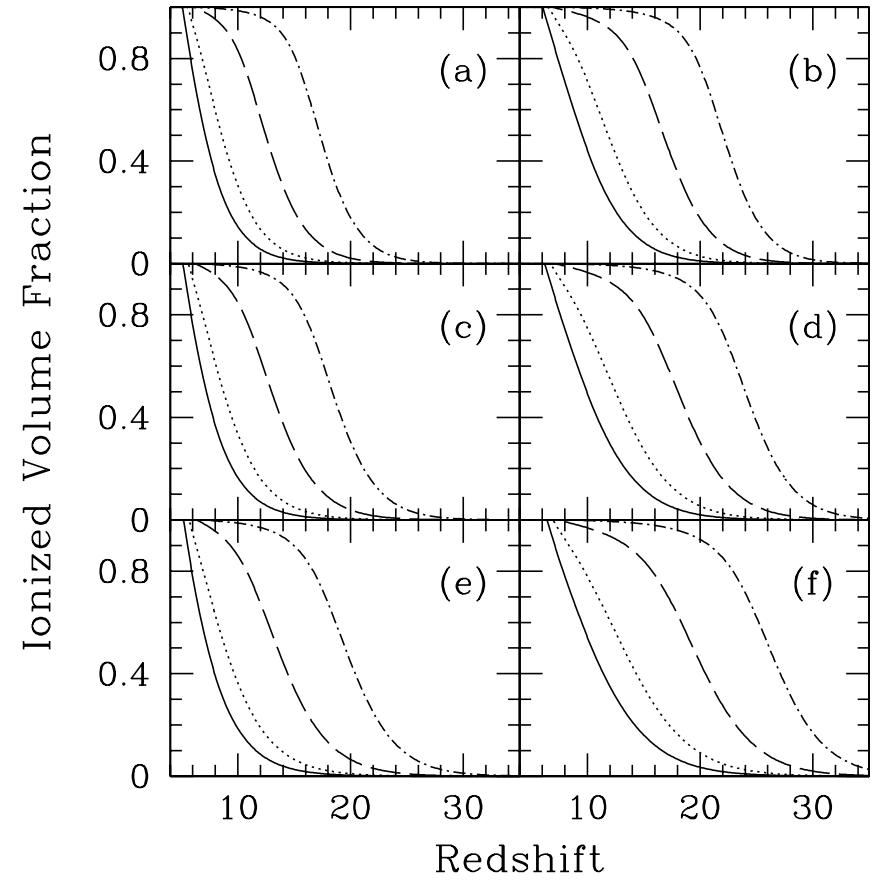

FIG. 3.-Reionization history of the universe for six cases: $(a)$ model $1, \alpha=0$; (b) model 1, $\alpha=1.5 ;(c)$ model 2, $\alpha=0$; $(d)$ model 2, $\alpha=1.5$; $(e)$ model 3, $\alpha=0 ;(f)$ model 3, $\alpha=1.5$. Within each panel, the lines indicate the reionization history for $\gamma=0$ (solid line), 1 (dotted line), 10 (dashed line), and 100 (dash-dotted line).

higher $\gamma$ result in higher optical depths because the IGM is reionized earlier.

\section{DISCUSSION}

For a fixed CDM halo population, the simplest model for the emissivity would say that the rate of photon emission per unit mass is constant for all halos in which cooling can take place, i.e., with $\sigma>\sigma_{\min }$. This model, using the emissivity per unit mass calibrated with the observational determination at $z=4$ and a clumping factor of 1 , predicts a redshift at which reionization ends of 5-7, in very good agreement with the observation of the appearance of the Gunn-Peterson trough at $z=6$. The emissivity history in this model shows a decline with redshift from $z=4$ to 6 , then a slight increase up to $z \sim 10$ as more population $\mathrm{B}$ halos contribute, and then a rapid decline beyond $z \sim 10-20$ as the number of all halos decreases.

Obviously, the reionization history should also depend on the clumping factor affecting the recombination rate. We have assumed a clumping factor of unity, and a higher clumping factor would delay the end of reionization. A moderate clumping factor of 2 or 3 would not greatly affect the result because the recombination time at the mean baryonic density is

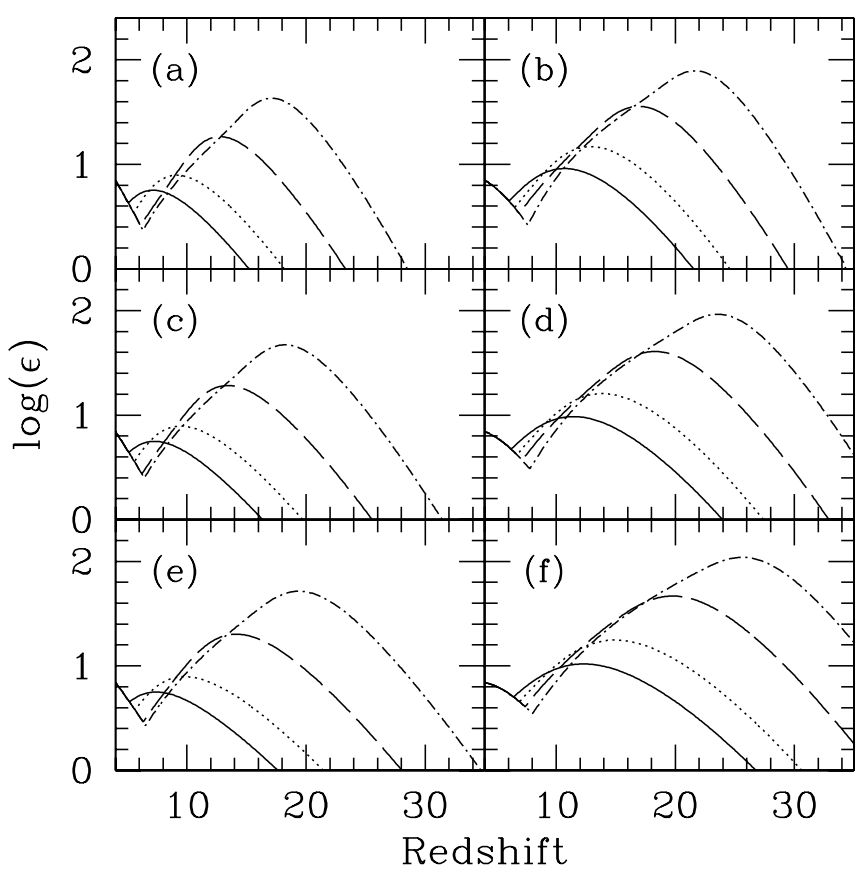

FIG. 4.-Logarithm of emissivity (ionizing photons per baryon per Hubble Time at $z=4$ ) vs. redshift. Panels and lines are as in Fig. 3

equal to the Hubble time at $z=6$ and the increase in $y(z)$ is relatively fast for the $\gamma=0$ models (Fig. 3, solid lines; this is simply due to the rapid increase in the mass fraction in collapsed halos shown in Fig. 1), so the number of recombinations is not very large. For example, a clumping factor of 3 for model 1 with $\alpha=1.5$ (Fig. $3 b$ ) delays the end of reionization from $z=6$ to 5 . The clumping factor is unlikely to be very large during reionization because high-density regions do not tend to be ionized until the cosmic ionizing background reaches a high intensity after reionization has ended and the mean free path of ionizing photons can be increased, as discussed in Miralda-Escudé et al. (2000) and Miralda-Escudé (2003). Although some authors have argued that high-density gas in low-mass halos could be ionized as reionization proceeds and increase the clumping factor, calculations of the hydrodynamic evolution of the gas in these halos by Shapiro \& Raga (2001) have shown that the gas expands rapidly after being ionized, giving rise to only a small number of recombinations. It is possible also to have a clumping factor lower than unity if most baryons are in highly self-shielded clumps, but this possibility is also unrealistic because at $z>6$ most of the baryons have not yet collapsed into halos and are therefore at densities on the order of the mean density. In summary, the uncertainty in the global recombination rate does not greatly affect the predicted epoch of the end of reionization.

TABLE 1

Model Optical Depths

\begin{tabular}{|c|c|c|c|c|c|c|}
\hline \multirow[b]{2}{*}{$\gamma$} & \multicolumn{2}{|c|}{ Model 1} & \multicolumn{2}{|c|}{ Model 2} & \multicolumn{2}{|c|}{ Model 3} \\
\hline & $\tau_{e}(\alpha=0)$ & $\tau_{e}(\alpha=1.5)$ & $\tau_{e}(\alpha=0)$ & $\tau_{e}(\alpha=1.5)$ & $\tau_{e}(\alpha=0)$ & $\tau_{e}(\alpha=1.5)$ \\
\hline $0 \ldots \ldots \ldots \ldots \ldots \ldots$ & 0.049 & 0.074 & 0.050 & 0.080 & 0.052 & 0.086 \\
\hline 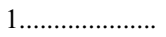 & 0.062 & 0.096 & 0.065 & 0.105 & 0.068 & 0.115 \\
\hline $10 \ldots \ldots \ldots \ldots \ldots$ & 0.101 & 0.153 & 0.108 & 0.170 & 0.116 & 0.190 \\
\hline $100 \ldots \ldots \ldots \ldots \ldots$ & 0.159 & 0.226 & 0.175 & 0.256 & 0.191 & 0.290 \\
\hline
\end{tabular}


Therefore, we have the remarkable result that the simplest model of a constant emissivity per unit mass in CDM halos, calibrated with the observed emissivity at $z=4$, roughly predicts the right epoch for the end of reionization.

This same model predicts an optical depth to Thomson scattering, $\tau_{e}$, of $0.05-0.09$, depending on $\alpha$ and on the allowed variations of the power spectrum parameters determining the amplitude of fluctuations on the small scales of the first halos. Although this is smaller than the value found by the WMAP mission in a CDM model fitted according to Spergel et al. (2003), $\tau_{e}=0.17 \pm 0.06$, it is not ruled out with the present error bar: the upper value of $\tau_{e}=0.09$ of our $\gamma=0$ models is consistent with this measurement at the $1.3 \sigma$ level. The likelihood function of this range of $\tau_{e}$ shown in Figure 8 of Spergel et al. (2003) is also seen not to be very small. Moreover, if the CDM model with a power-law primordial spectrum is imposed (instead of allowing a running spectral index), then the best fit yields $\tau_{e}=0.12 \pm 0.06$ (see Table 7 of Spergel et al.). The strong degeneracy of $\tau_{e}$ with the spectral index $n$ should also be noted in Figure 5 of Spergel et al., so for our model 1 with $n_{s}=0.96$ a value $\tau_{e}=0.06$ is consistent with the WMAP data at the $1-\sigma$ level.

In conclusion, there is no inconsistency between the WMAP results and the low value of $\tau_{e}$ predicted in a model in which the emissivity of low-mass halos at high redshift is no larger than the emissivity from halos at $z=4$ determined observationally. The smaller error bar for $\tau_{e}$ obtained by the analysis in Kogut et al. (2003) is puzzling because the addition of observational data and the use of parameterized models in Spergel et al. instead of the model-independent analysis in Kogut et al. should not result in an increased error.

If the high value of $\tau_{e}$ found by Kogut et al. (2003) is confirmed by future data, then the ionizing emission efficiency in the earliest low-mass halos to form in CDM is required to be much higher than in the sources present in the more massive halos at $z=4$. The required factor by which the efficiency must increase can be inferred from Table 1 . The increase in emissivity per unit mass is $\sim(1+2 \gamma)(1+z)^{\alpha}$ (see eq. [3]) for a typical halo contributing to the emissivity at high redshift with $\sigma \sim 5 \mathrm{~km} \mathrm{~s}^{-1}$. For the case $\alpha=0$, the factor $1+2 \gamma$ gives the increase in the rate of emission per unit mass, while for the case $\alpha=1.5$, the factor $1+2 \gamma$ gives the increase in the total energy per unit mass emitted in ionizing photons, taking into account the shorter Hubble time at higher redshift. Interpolating from Table 1 , we find that to reach $\tau_{e}=0.17$, the total ionizing energy per unit mass emitted by population $\mathrm{B}$ halos at $z \gtrsim 15$ needs to be larger by at least a factor of $1+2 \gamma \sim 10$ compared with that of the population A halos at $z=4$, and the rate of emission needs to be higher by a factor of $\sim 100$. For model 1 (which has a lower power spectrum amplitude and is more consistent with the Croft et al. 2002 measurement of the Ly $\alpha$ forest power spectrum), the energy emitted per unit mass needs to increase by a factor of $\sim 25$, and the emission rate per unit mass needs to increase by $\sim 300$.

Are these very high efficiencies for producing ionizing photons in the earliest low-mass halos physically realistic? The emissivity of population A halos at $z=4$ is about 60 photons per baryon per Hubble time, as obtained by dividing the mean emissivity $\epsilon_{4}$ of 7 photons per baryon per Hubble time (see $\S 2.3$ ) by the fraction of baryons in population A halos at $z=4$ (see Fig. 1). From the emission ratios mentioned above required to produce $\tau_{e}=0.17$, population $\mathrm{B}$ halos at $z \simeq 20$ would need to emit $\sim 1500$ and 600 photons per Hubble time for models 1 and 3, respectively. Since in these models with high optical depth the universe is reionized at $z \simeq 20$ over a time interval corresponding to $\Delta z \simeq 5$ (see Fig. 3), or one-quarter of a Hubble time, the total emission during this time interval coming from the first halos needs to be about 400 and 150 photons per baryon in population B halos for models 1 and 3 , respectively.

To compare this with the number of photons that can be produced by star formation, we use the models of Leitherer et al. (1999; see their Fig. 78) to find that for a Salpeter IMF with solar metallicity in the stellar mass range $1 M_{\odot}<M<$ $100 M_{\odot}, 6000$ ionizing photons per baryon are emitted. Most of the ionizing photons are produced by stars with $M>20 M_{\odot}$, and in the case of a Salpeter IMF with stars forming only in the range $20 M_{\odot}<M<100 M_{\odot}, 25,000$ photons per baryon can be emitted. Note that this number is equal to the ratio of the energy obtained by hydrogen fusion $(7 \mathrm{MeV})$ to the average energy of an ionizing photon $(20 \mathrm{eV})$ times the fraction of stellar mass that is fused to helium $(\sim 30 \%)$ times the fraction of energy emitted as ionizing photons $(\sim 25 \%)$. Very massive stars with zero metallicity can further increase this emission to $10^{5}$ photons per baryon (Tumlinson \& Shull 2000; Bromm et al. 2001; Schaerer 2002) because their convective cores during the main sequence include almost all the mass of the star (zerometallicity stars also have higher temperature than metal-rich ones during the main sequence, which increases the fraction of energy they emit in ionizing radiation but also increases the average energy of their ionizing photons). Thus, if $f_{*}$ is the fraction of the gas in a halo that can be converted to stars with $M>20 M_{\odot}$ and $f_{\text {esc }}$ is the fraction of ionizing photons that escape to the IGM, then an optical depth $\tau_{e}=0.17$ requires $f_{*} f_{\text {esc }}>10^{-2.4}$ and $10^{-2.8}$ for models 1 and 3 , respectively, for zero-metallicity stars and $f_{*} f_{\text {esc }}>10^{-1.8}$ and $10^{-2.2}$ for models 1 and 3 , respectively, for metal-enriched stars.

It is clear from these values that an optical depth $\tau_{e}=0.17$ can be produced with physically realistic models. Even $\tau_{e}=$ 0.3 is possible in model 3 with $\gamma=100$ and $\alpha=1.5$, which implies an emission of 3000 ionizing photons per baryon (or $f_{*} f_{\text {esc }}=10^{-1.5}$ for zero-metallicity stars) from the first starforming halos over the redshift range $25 \lesssim z \lesssim 32$. However, if a high optical depth is confirmed by future measurements, an explanation for the dramatic change in ionizing emission between the low-mass halos that formed the first stars and the more massive halos at lower redshift will need to be found.

A difficulty in making star formation very efficient in lowmass halos is that their shallow potential wells make them more vulnerable to losing their gas as a result of stellar winds, photoionization, and supernovae. The escape fraction might be higher than in more massive halos at lower redshift but only after gas has been ionized and expelled from the vicinity of stars, in which case the gas can easily escape from the halo. The typical halo where stars form at $z \simeq 20$ with velocity dispersion of $\sim 5 \mathrm{~km} \mathrm{~s}^{-1}$ and total mass of $10^{6} M_{\odot}$ (e.g., Yoshida et al. 2003a) has a gas mass of $\sim 2 \times 10^{5} M_{\odot}$ within a virialized radius of $\sim 200 \mathrm{pc}$, and cooling by molecular hydrogen leads to the formation of a dense central gas clump containing only $\sim 1 \%$ of the gas mass. The rest of the gas must take more than 1 halo dynamical time $\left(\sim 10^{7.5} \mathrm{yr}\right)$ to reach the center and cool before it has any possibility of forming stars, by which time supernovae would have taken place. The hydrodynamic simulations of these first star-forming halos (Abel et al. 2002; Bromm et al. 2002) suggest that the first stars may all be very massive. If the $\sim 2000 M_{\odot}$ of the core of cooled gas can efficiently form one or a few massive stars and most of the emitted ionizing photons escape, one may barely be able to 
achieve the fraction $f_{*} f_{\text {esc }} \sim 10^{-2.5}$ required for $\tau_{e}=0.17$. After the formation of these stars, the supernovae explosions would expel the remaining gas from the halo (Bromm et al. 2003).

In halos of higher mass (with $\sigma$ of $10-30 \mathrm{~km} \mathrm{~s}^{-1}$ ), a larger number of supernovae would be required to expel the gas. Nevertheless, the effects of photoionization and supernova explosions should still self-regulate the rate of massive star formation, and it is not clear why the earliest galaxies at high redshift could have higher values of $f_{*} f_{\text {esc }}$ than the more massive galaxies formed at lower redshift. While the mean gas density in galaxies should increase with redshift, increasing gas cooling rates, the shallower potential wells of low-mass halos allow for gas dispersal after a smaller fraction $f_{*}$ of gas has turned into massive stars.

Another possibility is that black holes formed by the first massive stars are able to accrete gas. Gravitational accretion can produce radiation with an efficiency $\sim 20$ times greater than stellar fusion (and stars generally fuse only a fraction of their mass). In this case, requiring a fraction as low as $\sim 10^{-3.5}$ of the halo gas to accrete onto a central black hole could produce the $\sim 300$ photons per baryon in halos required for $\tau_{e}=0.17$ if the fraction of the radiation energy that the accreting gas emitted as ionizing photons were $\sim 20 \%$. However, whether one speculates about a high efficiency of massive star formation or of gas accretion onto black holes, the question that remains unanswered is why such high efficiency in producing ionizing radiation would occur only in the first halos at high redshift and not in similar or more massive halos at a later epoch.

If it were nevertheless possible to produce these high emissivities when the first stars formed, a second possible problem of an early reionization might be that the high emissivity leads to a rapid end of reionization, in contradiction to the presence of the Gunn-Peterson trough at $z=6$. Our results suggest a way to solve this particular problem: as seen in Figure 3, once the fraction of the IGM that is ionized becomes close to unity, the emissivity can be decreased because star formation in population B halos is suppressed. This can regulate the rate of emission to a level that just balances recombinations, and it is only when the more massive population A halos reach a critical abundance that the emissivity can rise again, completing reionization and causing the fast increase in the mean free path of ionizing photons that clears the GunnPeterson trough. Thus, in all our models the end of reionization occurs between redshift 5 and 8, even though the models with high $\gamma$ can ionize half of all the volume as early as $z \simeq 20$. In the models with the earliest reionization in Figure 3, the redshift for the end of reionization could easily be lowered to fit the observed value of 6 by moderately increasing the clumping factor after $y$ is close to unity or by increasing the value of $\sigma_{0}$ that separates our populations A and B.

Another possible problem of models with highly efficient star formation at high redshift is that any low-mass stars that were made together with the massive stars that caused the ionization should be visible today in dwarf galaxies. A large number of dark matter satellites that are not observed are predicted to exist around galaxies such as the Milky Way (e.g., Klypin et al. 1999; Moore et al. 1999). The various ideas that have been proposed to explain this absence of numerous small satellites within the CDM model context generally imply the suppression of star formation in low-mass halos at high redshift because of the effects of reionization and stellar winds (e.g., Bullock et al. 2000; Stoehr et al. 2002; Zentner \& Bullock
2003; Somerville et al. 2003). The high efficiency of massive star formation in these same halos required for accounting for a high $\tau_{e}$ would then imply that practically no low-mass stars could be formed while very massive stars were forming in large numbers, which appears unlikely.

Note that models with high $\gamma$ have an emissivity history that is double peaked: there is a first maximum due to population B halos; then the emissivity declines as most of the IGM is ionized, and a second maximum is then caused by population A halos. The possibility of a double-peaked history of star formation due to the effect of reionization raising the Jeans mass was proposed by Couchman \& Rees (1986) and Shapiro et al. (1994). However, this does not necessarily imply a "double reionization," as other authors have argued (Cen 2003; Wyithe \& Loeb 2003). The fraction of ionized volume, $y(z)$, rises monotonically with time, and it is only stalled by the suppression of the star formation due to ionization, in a selfregulated process. A double reionization in which the universe recombines again after having been ionized would require a long delay of the feedback ionization effects on the star formation rate. If this delay were present, the suppression of star formation might start to operate only after stars have already emitted more than enough photons to ionize all the universe; later, when the IGM recombines again, star formation might not resume at the same level for some time, allowing for partial recombination to occur. This feedback delay might be caused by a reservoir of gas in population $\mathrm{B}$ halos that can keep forming stars after the surrounding low-density medium is ionized and by the finite time required for new recombined and cooled gas to accrete in the population B halos after the IGM recombines again, but it is not clear whether this would be sufficient to cause the global instability in the star formation rate that would be required for a double reionization.

Finally, comparing our results with other recent work, we note that other authors (e.g., Wyithe \& Loeb 2003; Haiman \& Holder 2003; Ciardi et al. 2003; Somerville \& Livio 2003; Sokasian et al. 2003; Cen 2003) have generally emphasized that the central value of the $W M A P$ result $\tau_{e} \simeq 0.17$ can be obtained in CDM models with a physically allowed emissivity of ionizing photons. Although we agree with this conclusion, we note that a drastic change in the emissivity per unit mass of star-forming halos from $z \gtrsim 15$ to 4 is required. For example, Ciardi et al. (2003) find, using values close to those of our model 3 , that $\tau_{e}=0.16$ can be reached in models in which the dominant emission comes from halos corresponding roughly to our population A, but the emissivity they assume would vastly overpredict the intensity of the ionizing background at $z=4$. At the same time, models with no such change in the emissivity yield a lower optical depth that is still within the plausible error range of the $W M A P$ result. Our approach is closest to that of Chiu et al. (2003), who use an ionizing emission efficiency chosen to yield reasonable models for the ionizing background, and our conclusions are generally in agreement with theirs.

\section{CONCLUSION}

The first detection of the Thompson optical depth to the CMB by the WMAP mission introduces a new era in the study of reionization. The observations of the Gunn-Peterson absorption in the highest redshift quasars, which are sensitive to the time when reionization ended and the mean free path of ionizing photons increased most rapidly, can be confronted with the total ionized column to the surface of last scattering, which is sensitive to the epoch when about half the baryons in 
the universe were ionized. The simplest assumption we can make about the emissivity is that it is roughly constant in all halos in which gas can cool. When we calibrate the emissivity to the upper limit allowed by observations at $z=4$, we find that the end of reionization is close to $z=6$, as suggested by observations, but that the optical depth is smaller than 0.09 . To obtain an optical depth as high as $\tau_{e}=0.17$ (the central value of the WMAP result), an increase with redshift in the rate of ionizing emission per unit mass in collapsed halos of a factor of $\sim 100-300$ from $z=4$ to $\sim 20$ (depending on the CDM power spectrum model) is required.

Further progress on our understanding of the reionization epoch will come from, among other things, an increased accuracy in the measurement of $\tau_{e}$, as well as from more robust measurements of the power spectrum on small scales from Ly $\alpha$ forest observations. The discovery of quasars at $z>6$ and the search for any small fraction of Ly $\alpha$ transmitted flux in gaps along their Gunn-Peterson troughs (White et al. 2003) may also allow us to test whether the fraction of the ionized medium was close to unity for a wide redshift range above $z=6$ (as in our models with high $\gamma$ in Fig. 3) or whether it increased rapidly over a short redshift range. If an optical depth as high as the central value of the WMAP measurement is confirmed, then either a physical explanation for the high efficiency of the ionizing emissivity of the first objects will need to be found or some more fundamental modification of the CDM model, such as non-Gaussian fluctuations on small scales (e.g., Avelino \& Liddle 2004), may be implied.

We thank James Bullock for useful discussions and the use of his code. This work was supported in part by NSF grant NSF-0098515.
Abel, T., Bryan, G. L., \& Norman, M. L. 2002, Science, 295, 93

Avelino, P. P., \& Liddle, A. R. 2004, MNRAS, 348, 105

Barkana, R. 2002, NewA, 7, 85

Bennett, C. L., et al. 2003, ApJS, 148, 1

Bromm, V., Coppi, P. S., \& Larson, R. B. 2002, ApJ, 564, 23

Bromm, V., Kudritzki, R. P., \& Loeb, A. 2001, ApJ, 552, 464

Bromm, V., Yoshida, N., \& Hernquist, L. 2003, ApJ, 596, L135

Bullock, J. S., Kravtsov, A. V., Weinberg, D. H. 2000, ApJ, 539, 517

Cen, R. 2003, ApJ, 591, L5

Chiu, W. A., Fan, X., \& Ostriker, J. P. 2003, ApJ, 599, 759

Ciardi, B., Ferrara, A., \& White, S. D. M. 2003, MNRAS, 344, L7

Colless, M., et al. 2001, MNRAS, 328, 1039

Couchman, H. M. P., \& Rees, M. J. 1986, MNRAS, 221, 53

Croft, R. A. C., Weinberg, D. H., Bolter, M., Burles, S., Hernquist, L., Katz, N., Kirkman, D., \& Tytler, D. 2002, ApJ, 581, 20

Efstathiou, G. 1992, MNRAS, 256, P43

Eisenstein, D. J., \& Hu, W. 1999, ApJ, 511, 5

Fan, X., Narayanan, V. K., Strauss, M. A., White, R. L., Becker, R. H., Pentericci, L., \& Rix, H.-W. 2002, AJ, 123, 1247

Gnedin, N. 2000, ApJ, 535, 530

Gunn, J. E., \& Peterson, B. A. 1965, ApJ, 142, 1633

Haiman, Z., \& Holder, G. P. 2003, ApJ, 595, 13

Heap, S. R., Williger, G. M., Smette, A., Hubeny, I., Sahu, M., Jenkins, E. B., Tripp, T. M., \& Winkler, J. N. 2000, ApJ, 534, 69

Holder, G., Haiman, Z., Kaplinghat, M., \& Knox, L. 2003, ApJ, 595, 13

Klypin, A., Kravtsov, A. V., Valenzuela, O., \& Prada, F. 1999, ApJ, 522, 82

Kogut, A., et al. 2003, ApJS, 148, 161

Leitherer, C., et al. 1999, ApJS, 123, 3

Madau, P., Haardt, F., \& Rees, M. J. 1999, ApJ, 514, 648

\section{REFERENCES}

Miralda-Escudé, J. 1998, ApJ, 501, 15

2003, ApJ, 597, 66

Miralda-Escudé, J., Haehnelt, M., \& Rees, M. J. 2000, ApJ, 530, 1

Moore, B., Ghigna, S., Governato, F., Lake, G., Quinn, T., Stadel, J., \& Tozzi, P. 1999, ApJ, 524, L19

Navarro, J., \& Steinmetz, M. 1997, ApJ, 478, 13

Press, W. H., \& Schechter, P. 1974, ApJ, 187, 425

Schaerer, D. 2002, A\&A, 382, 28

Seljak, U., McDonald, P., \& Makarov, A. 2003, MNRAS, 342, L79

Shapiro, P. R., Giroux, M. L., \& Babul, A. 1994, ApJ, 427, 25

Shapiro, P. R., \& Raga, A. C. 2001, Rev. Mexicana Astron. Astrofis. Ser. Conf., 10,109

Sheth, R. K., \& Tormen, G. 1999, MNRAS, 308, 119

Sokasian, A., Abel, T., Hernquist, L., \& Springel, V. 2003, MNRAS, 344, 607

Somerville, R. S., Bullock, J. S., \& Livio, M. 2003, ApJ, 593, 616

Somerville, R. S., \& Livio, M. 2003, ApJ, 593, 611

Spergel, D. N., et al. 2003, ApJS, 148, 175

Stoehr, F., White, S. D. M., Tormen, G., \& Springel, V. 2002, MNRAS, 335, L84

Storrie-Lombardi, L., McMahon, R. G., Irwin, M. J., \& Hazard, C. 1994, ApJ, 427, L13

Thoul, A. A., \& Weinberg, D. H. 1996, ApJ, 465, 608

Tumlinson, J., \& Shull, J. M. 2000, ApJ, 528, L65

White, R. L, Becker, R. H., Fan, X., \& Strauss, M. A. 2003, AJ, 126, 1

Wyithe, J. S. B.., \& Loeb, A. 2003, ApJ, 588, L69

Yoshida, N., Abel, T., Hernquist, L., \& Sugiyama, N. 2003a, ApJ, 592, 645

Yoshida, N., Sokasian, A., Hernquist, L., \& Springel, V. 2003b, ApJ, 598, 73

Zentner, A. R., \& Bullock, J. S. 2003, ApJ, 598, 49 